\title{
Patrones de aprovechamiento del saíno sin collar Tayassu Pecari (Artiodactyla: Tayassuidae) en seis municipios del departamento del Chocó, Colombia
}

\section{Model of advantage of Tayassu Pecari (Artiodactyla: Tayassuidae) in six municipalities of the department of the Choco, Colombia}

\author{
David Garrido Serrano, MDV'1, Tatiana Palacios Martínez, Ing ${ }^{1}$, \\ Leison Palacios Mosquera, Biol ${ }^{2}$
}

\section{RESUMEN}

Con el fin de aportar al conocimiento tradicional del Pecari tajacu en la zona centro del Chocó, se realizó este estudio que ofrece información del uso popular, las formas de aprovechamiento del tatabro sin collar y la forma de entender esta actividad por parte de los pobladores de la región. Los datos se obtuvieron, a través de encuestas realizadas a personas residentes en los municipios de Quibdó, Atrato, Unión Panamericana, Cantón de San Pablo, Medio Baudó y Cértegui. Se encontraron cuatro categorías de uso, de las cuales la alimentación es la más importante, seguida del uso artesanal. Aún se conservan los métodos de cacería tradicionales siendo los más empleados el machete (25\%), el perro (24\%) y la escopeta (23\%). El uso de la fauna silvestre está muy relacionado con el conocimiento empírico y tradicional de las comunidades locales, que considera el tatabro como una importante fuente proveedora de proteína animal; se identificó además una disminución en el tamaño de las poblaciones y una reducción en la frecuencia de captura.

Palabras clave: Saíno; Fauna silvestre; Aprovechamiento; Biodiversidad.
1. Investigador, Instituto de Investigaciones Ambientales del Pacífico(IIAP), Quibdó, Colombia. e-mail: dagase55@gmail.com tatipama2008@hotmail.com

2. Grupo de Investigación y Manejo de Fauna Silvestre del Chocó, Universidad Tecnológica del Chocó, Quibdó Colombia. e-mail:lepamo8@gmail.com

Recibido: 26 de marzo, 2010

Aceptado: 22 de abril, 2010

\begin{abstract}
With the purpose of to contribute to the traditional knowledge of the Pecari tajacu in the zone center of Choco this study was realised that offers information of the traditional use and the forms of advantage of tatabro without necklace and the form to understand this activity on the part of the settlers of the region. The data were obtained, through surveys realised to resident people in the municipalities of Quibdo, Atrato, Union Panamericana, Canton de San Pablo, Medio Baudo and Certegui. Four categories of use were reported, of which the feeding is most important, followed of the artisan use. The traditional methods of hunting are even conserved being used the machete (25\%), the dog (24\%) and the gun (23\%). The use of the wild fauna this closely related to the empirical and traditional knowledge of the local communities and considers to tatabro like an important source protein supplier animal, in which they identify in addition a diminution in the size to the populations and a reduction in the capture frequency.
\end{abstract}

Keywords: Saino; Wild fauna; Advantage; Biodiversity. 


\section{INTRODUCCIÓN}

Colombia como uno de los países con mayor biodiversidad en el mundo, posee un alto potencial para el comercio de bienes y servicios provenientes de la vida silvestre a partir de estrategias de aprovechamiento sostenible; dentro de estas estrategias se han desarrollado proyectos de cría en cautiverio que conduzcan al mantenimiento y conservación de las especies de fauna. La sobreexplotación o aprovechamiento no sostenible de especies silvestres de fauna para el consumo doméstico o la comercialización, tiene graves efectos sobre la biodiversidad, como la erosión genética, la reducción de los tamaños de poblaciones y la vulnerabilidad frente a procesos de extinción (Mancera-Rodríguez y Reyes-García 2008).

Gran parte del aprovechamiento de especies de fauna silvestre, en su mayoría mamíferos, con fines alimenticios a través de la actividad de cacería, está ligado a las comunidades humanas habitantes en el departamento del Chocó que hacen uso de los ecosistemas y de sus componentes de diferentes maneras. En este caso, existen diferentes modalidades de uso de especies, que reflejan diferencias locales, sociales, económicas, culturales (Ojasti 1993). Este es uno de los aspectos más importantes de la conservación de la biodiversidad, que genera un impacto significativo en las poblaciones animales y en las poblaciones humanas.

La carne de monte producto de la caza es un recurso económico importante que usan tradicionalmente las comunidades rurales, y que de ser manejada de forma adecuada, podría proporcionar beneficios económicos a largo plazo a estas comunidades, y al mismo tiempo podría ayudar a conservar las especies silvestres más amenazadas. En la región del Chocó, a pesar de existir algunos estudios sobre la fauna silvestre, es muy limitado el conocimiento que se tiene sobre el uso, manejo, aprovechamiento y conservación de la misma; pero al mismo tiempo no se cuenta con suficiente información biológica de las especies ni socio-económica de las comunidades que las aprovechan, de tal manera que se permita el diseño de un adecuado plan de manejo sostenible de la biodiversidad de la región. Se informan muy pocos trabajos, entre los que se destacan los realizados por Cuesta-Ríos et al. (2007) donde se caracterizaron los patrones de uso de la fauna silvestre en el área de influencia de la Estación Ambiental Tutunendo (EAT), Quibdó y Valderrama Ballesteros (2009), quien estudia el saíno (Pecari tajacu) a partir del conocimiento local y la observación de sus huellas en el territorio de El Valle, Chocó, Colombia.

Este estudio brinda información del uso tradicional y las formas de aprovechamiento del tatabro sin collar suministrada por los pobladores locales y la forma de entender la actividad de caza en la región, buscando aportar al conocimiento tradicional del P. tajacu en la zona centro del Chocó.

\section{ÁREADE ESTUDIO}

El departamento del Chocó tiene una extensión de 46.530 $\mathrm{km}^{2}$, que corresponden a $4 \%$ del total del territorio colombiano; está localizado a los $4^{\circ} 10^{\prime}$ y a $\operatorname{los} 8^{\circ} 10^{\prime}$ de latitud norte y entre los $76^{\circ}$ y $78^{\circ}$ de longitud oeste; es la única región de América con costas sobre dos océanos. Se encuentra en el noroeste del país, en la región del Pacífico colombiano, entre las selvas del Darién y las cuencas de los ríos Atrato y San Juan, entre la cordillera Occidental y el océano Pacífico(IGAC 2006). Se caracteriza por su alta pluviosidad oscilante entre $8000-11.000$, una temperatura promedio de $28^{\circ} \mathrm{C}$, su topografía variable va desde un metro sobre el nivel del mar (zona costera) hasta los 2800 msnm (Cerro del Torrá, San José del Palmar y Carmen de Atrato).

Su población está conformada en un $90 \%$ por afro-descendientes en diferentes grados de mestizaje; los grupos indígenas equivalen a 6\%: Cunas, Emberas y Waunanas (se encuentran para todo el departamento 82 resguardos indígenas, de los cuales seis comparten con el departamento del Valle del Cauca) y los mestizos corresponden a $4 \%$ del total de la población(CODECHOCO 2009).

Las diferentes etapas de este estudio se realizaron en los municipios de Quibdó, Atrato, Unión Panamericana, Cantón de San Pablo, Medio Baudó y Cértegui en el departamento del Chocó, Colombia, que se agruparon en tres zonas conformadas por dos municipios cada una, en el orden como aparecen antes citados, para efecto de facilitar los análisis.

\section{MATERIALES Y MÉTODOS}

Desarrollo de entrevistas. Entre los meses de octubre y noviembre de 2009 en el marco del proyecto Plan de manejo para la conservación in situ de los puercos de monte (Tayassu tajacu y T. pecari) y los osos perezosos (Bradypus variegatus y Choloepus hoffmanni), en los municipios de Quibdó, Atrato, Unión Panamericana, Cantón de San Pablo, Medio Baudó y Cértegui en el departamento del Chocó, Colombia, se obtuvo la información mediante el desarrollo de entrevistas semiestructuradas y charlas con cazadores y demás habitantes de la zona acerca de los puercos de monte, talleres y reuniones con convocatoria abierta a la población en general de cada comunidad visitada. Las encuestas se desarrollaron siguiendo el protocolo tomado de CODECHOCO-UTCH(2009), con algunas modificaciones. Para un mejor manejo de la información, las encuestas tuvieron como base las siguientes unidades temáticas, propuestas por Orozco (2001): información personal del entrevistado, historia natural y biología de las especies, abundancia y distribución, datos sobre el estado y la mortalidad de la especie, cultura y tradición, vigilancia, comercialización y cría. 
Bioetnia Volumen 7 № 2 (julio-diciembre), 2010

Tabla 1

Número de cazadores encuestados por localidad

\begin{tabular}{|c|c|c|c|}
\hline Zonas & Municipios & Localidades & Número de cazadores \\
\hline \multirow[t]{4}{*}{ I } & Quibdó & Tutunendo & 5 \\
\hline & & Pacurita & 1 \\
\hline & Atrato & Yuto & 3 \\
\hline & & Samurindó & 2 \\
\hline \multirow[t]{4}{*}{ II } & Unión Panamericana & Salero & 1 \\
\hline & & Ánimas medio & 1 \\
\hline & Cantón de San Pablo & Managrú & 1 \\
\hline & & Santiago & 1 \\
\hline \multirow[t]{4}{*}{ III } & Medio Baudó & Puerto Meluk & 10 \\
\hline & & Curundó La Banca & 6 \\
\hline & Cértegui & Variante & 5 \\
\hline & & La Toma & 3 \\
\hline Total & & & 39 \\
\hline
\end{tabular}

Esto permitió elaborar una guía de entrevista, con los temas mencionados a manera de resumen; las encuestas fueron semi-estructuradas y flexibles, con preguntas abiertas que facilitaban una respuesta amplia y en los términos propios del entrevistado, mediante una triangulación que consistió en juntar datos de distintas fuentes sobre un mismo tema. Durante el desarrollo de las entrevistas se evitó la adquisición de información distorsionada que podían dar los habitantes (Gelilfus 1998), lo que permitió obtener información más confiable.

Las entrevistas se organizaron y estudiaron mediante análisis descriptivos para obtener una mejor información acerca del estatus de la especie por parte de las comunidades locales. En este caso, se identificó la importancia de la fauna para los habitantes, los respectivos beneficios y perjuicios que ofrece, el conocimiento de los pobladores sobre acciones de conservación de las especies y la percepción de los mismos sobre el estado poblacional de la fauna; se reconocieron las especies abundantes y menos abundantes y se identificaron las posibles causas de estas situaciones, todo esto con el fin de determinar la percepción, conocimiento empírico y tradicional que tienen los pobladores sobre el $T$. pecari.

\section{RESULTADOSYDISCUSIÓN}

De acuerdo con la información levantada con los habitantes de las zonas, la época del año en la que se observa un mayor número de individuos, es en verano (76\%), mientras que un $24 \%$ afirma que todo el año se observan indicios del tatabro sin collar. Esta época de verano hace referencia al período de bajas lluvias comprendido entre los meses de diciembre, enero y marzo, que constituyen la mejor temporada para la cacería de los puercos de monte, debido tal vez a que se presenta mayor disponibilidad de recurso trófico para las especies y un incremento de su actividad reproductiva.

Entorno socio-cultural de los cazadores. Se identificaron 39 cazadores (informantes claves), que practican la cacería de la especie $T$. pecari, en doce localidades de las zonas de estudio (Tabla 1), quienes además combinan ésta con otras actividades socioeconómicas, como la agricultura, la tala selectiva de madera y la minería. La totalidad de las entrevistas se aplicó a hombres de las comunidades porque son ellos quienes se dedican a la cacería a pesar de no ser ésta su principal actividad. Montero (2004), afirma que la cacería es una actividad masculina que brinda reconocimiento y que quizá las «mujeres no la practican porque no es costumbre». Así ellas se vuelven indispensables para mantener informados a los hombres de la situación en la labranza, pero también para la preparación de la presa, pues ella es la encargada de esta tarea. Aunque ellas no van de cacería aquieren el conocimiento sobre los animales de monte por medio del padre o del esposo; el sistema que se emplea, el modo y las circunstancias del proceso de cacería se comparten con todos los miembros del hogar. El hombre de estas zonas comparte el conocimiento que tiene sobre los animales silvestres con la familia, es él quien sabe más sobre esta parte del sistema biofísico porque los captura y se encarga de transmitir el conocimiento a través del tiempo entre diferentes generaciones.

Instrumentos y técnicas empleadas por los pobladores 
de la zona para la cacería. A pesar de que los cazadores manifestaron no realizar la caza de puercos de monte de manera constante, aún se conservan los métodos de cacería tradicionales, siendo los más importantes: el perro (24\%), la escopeta(23\%), el lazo(12\%), el hacha $(11 \%)$ y el machete $(25 \%)$, éste último es un elemento imprescindible durante la actividad, pues también lo utiliza el cazador para abrir senderos o caminos que lo conducen donde está el animal rastreado por el perro, además sirve para rematar las presas heridas por la escopeta u otro elemento (Gráfica 1).

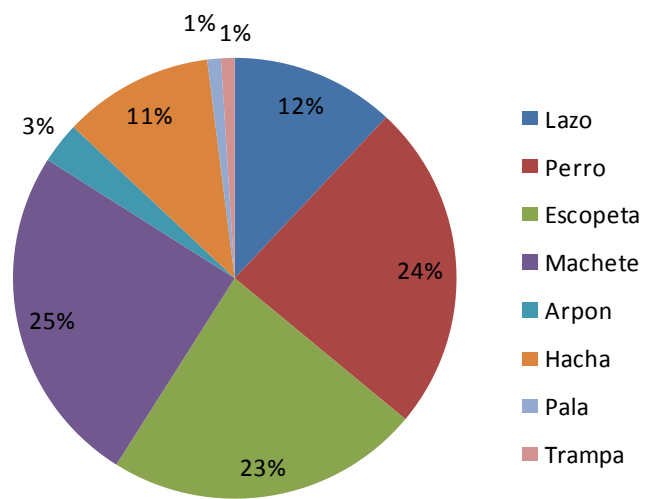

\section{Gráfica 1. Principales métodos de cacería utilizados para la captura de los puercos de monte.}

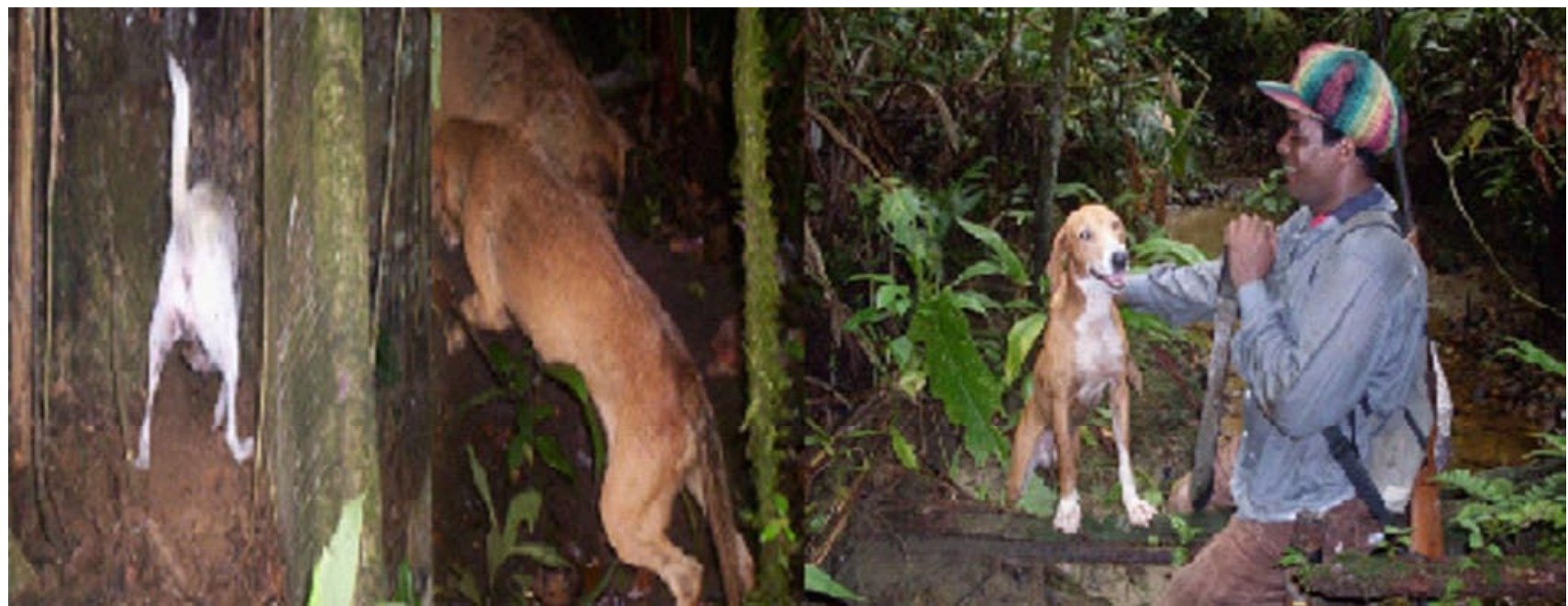

Figura1. Técnica utilizando el perro para la cacería de los puercos de monte en las comunidades de las zonas de estudio.

Elperro es la técnica más usual al momento de realizar la cacería actividad para la que los cazadores los entrenan desde los primeros años de su vida; este animal se considera además el complemento ideal para las otras técnicas como lazo, escopeta o machete, por su destreza que se refleja cuando rastrea la huella de la presa y la obliga a huir hasta su madriguera, en donde es atrapada por el cazador. Se pudo evidenciar que al final de cada faena de cacería, es común premiar al perro dándole algunas de las partes del animal capturado, como la cabeza, patas y/o visceras, posterior a ello se le da un baño con un combinado de enjuague de las visceras y pelo quemado del animal cazado, o sólo utilizan un sobijo con pelo quemado o plantas amasadas; esto con el fin de mejorar su habilidad para cazar (Figura 1).

La escopeta es un arma de fuego de cacería tradicional, que se utiliza cuando se observa alguna especie que resulta blanco fácil. La escopeta utilizada por los cazadores por lo general es un «chipún» (arma de fuego artesanal de calibre 12), elaborada en la región con madera nativa como carrá o costillo, que consta de un tubo metálico soldado en la parte de atrás con una grasera y un tornillo de enrosque en su parte proximal, que luego se monta y adapta a una caja de madera o culata.

El lazo, es un sistema tradicional de caza empleado para capturar la presa viva; consta de una vara de madera resistente y flexible, que se entierra unos centímetros, del otro extremo se fija un pedazo de cuerda al que se le hace un aro con un nudo corredizo colocado alrededor de un hueco construido en el suelo, cubierto por encima con tirillas delgadas de palmas, hojarascas y tierra, de tal manera que la trampa no sea visible para el animal; al pisarla ésta se dispara hacia arriba, cerrando el aro y atrapando al individuo que queda colgando bien sea, de la mitad de su cuerpo o de una de sus extremidades. El tatabro por ser un animal agresivo muchas veces termina cortando parte de la cuerda para escapar, por lo que los cazadores optan por cubrir el extremo del lazo con un 


\section{Bioetnia Volumen 7 No 2 (julio-diciembre), 2010}

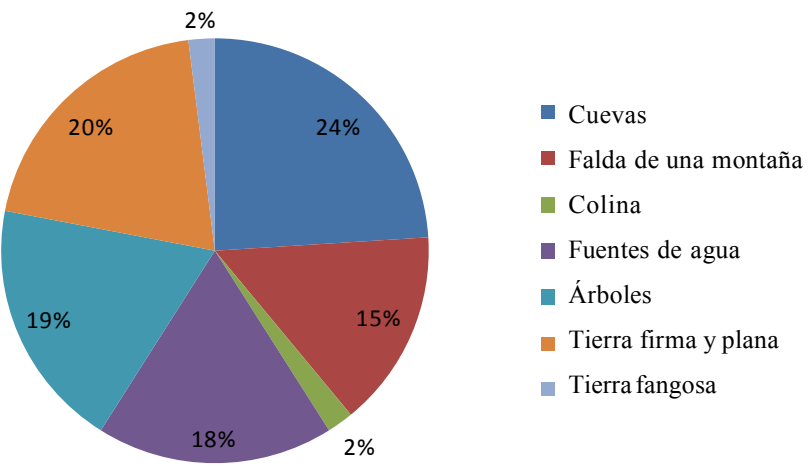

\section{Gráfica 2. Representatividad de sitios más aptos para la cacería de los puercos de monte, en localidades del área de estudio del proyecto.}

pedazo de guadua o de un tubo de PVC, que le imposibilita partirlo (CODECHOCOUTCH2009).

Lugares y jornada del día preferida para la cacería. Los lugares de captura más frecuentes son en primer orden las cuevas de tierra firme y plana, seguidas por raíces de árboles, fuente de agua y faldas de montaña. En cuanto a la jornada del día preferida para realizar la cacería, se encontró que prefieren las horas de la mañana, quizás por ser las horas que utilizan los animales para descansar y acicalarse, después de las grandes jornadas de alimentación nocturna (Gráfica 2).

Uso del tatabro sin collar. En total se identificaron para las zonas cuatro formas de utilización de la fauna y se determinó que el principal uso dado a este recurso producto de la cacería, es la alimentación, pues a través de ésta los pobladores aseguran una fuente proveedora de proteínas animal y la generación de recursos económicos.

Alimentación. Es el principal uso o destino de la cacería de los puercos de monte ( $65 \%$ de los casos), constituye la posibilidad de obtener proteína animal como fuente de alimento, lo que justifica que éste sea el uso de mayor ocurrencia en elárea de estudio. Zapata-Ríos et al. (2006) afirman que la caza de fauna silvestre con fines alimenticios (cacería de subsistencia) afecta, en forma negativa, las poblaciones animales de ciertas especies por la presión que ejercen las comunidades sobre éstas.

Las mujeres son las encargadas de preparar y repartir las presas en la familia, ellas aseguran que su preparación consiste en despojar al animal de la piel o cuero, eviscerarlo y hacer los cortes de las diferentes piezas de carne, lavarlo con agua caliente o hervida y después realizar su preparación ya sea sudado, sancochado, frito o guisado, previo ahumado, que le permite mejorar su sabor y conservación, tal como lo informan otros estudios como el de Guerrero y Ballesteros (2006).

A pesar del fuerte olor segregado por la glándula de los puercos de monte, los cazadores afirman que su carne tiene buen sabor como otros animales silvestres, lo que los motiva a alimentarse de la carne de esta especie sin ninguna limitante o restricción. Jiménez (2003), afirma que los animales de monte son importantes porque adornan el paisaje; además la carne tiene mejor sabor y es muy saludable dado que se alimentan de fuentes naturales como semillas, frutos y raíces entre otros, lo que hace que la carne sea especial y diferente.

Por tradición, el uso alimenticio dado a la fauna silvestre por parte de las comunidades, está ligado al aprovechamiento selectivo de especies que tiene un alto valor económico, estas especies aportan la mayor parte de la biomasa cazada, como son los mamíferos grandes y medianos entre las que encontramos al $T$. pecari. Tejada et al. (2006) afirman que especies como ésta son las más importantes dentro de la dieta de los pueblos.

Comercio. La caza con fines comerciales en la actualidad no es una actividad usual en las comunidades del área de estudio, según lo que manifiestan los pobladores; antes esta actividad era más frecuente por la abundancia de estas especies y la escases de otras fuentes de proteína animal que hoy en día son de fácil consecución. De otra parte la carne que queda después del consumo familiar, entra en un complejo sistema económico interno de distribución: se regala, por lo general dentro del circuito familiar, se presta a un compañero o amigo cazador hasta que se devuelve la misma cantidad $\mathrm{y} / \mathrm{o}$ se intercambia por otros productos o se vende en algunos casos, todo esto con el fin de obtener algunos recursos económicos que conduzcan a satisfacer sus necesidades, bien sea a través de la compra o del intercambio por otros productos que complementan la dieta familiar.

Con respecto a la comercialización de la carne del tatabro, se estableció, que ésta se realiza dentro de las mismas comunidades y que sus valores oscilan entre $\$ 3.000$ y $\$ 7.000$ por libra, valores que fluctúan dependiendo de la comunidad y el lugar donde se compra y/o se venda; el valor más alto dentro de la misma comunidad se presentó en las localidades de Tutunendo y Pacurita, mientras que el costo de la libra fuera de la comunidad osciló entre $\$ 8.000$ y $\$ 10.000$. En seis de las localidades estudiadas, no se realiza esta actividad.

Artesanía. Para los habitantes de la zona de estudio, el uso artesanal que se le da a los productos y/o subproductos de la cacería del tatabro, no es muy común; sólo en la comunidad de Puerto Meluk y Cantón de San Pablo, mencionaron haberles dado uso artesanal, empleando la piel o cuero del saíno en la construcción de sillas y tamboras.

Zoocría. Es una actividad poco usual en estas comunidades, porque los cazadores no ven viable esta práctica, quizás 
por la poca rentabilidad que puede tener el hecho de criar un animal por un tiempo indeterminado y desconocido para ellos. Además, al desconocimiento de la biología y manejo de la especie, se suma el riesgo de las fugas, agresiones o mordeduras a integrantes del hogar, enfermedades y muerte de los ejemplares, que para estos casos por lo general serealiza con animales juveniles o crías. Ojasti (2000), afirma que la producción de los animales silvestres en cautiverio suele ser más costosa que la de los animales domésticos, porque estos son más mansos, manejables y productivos, gracias al largo período de domesticación; sólo en la comunidad de Puerto Meluk, manifestaron haber tenido este tipo de animales en cautiverio.

Medicina tradicional, ritos y creencias mágicas y religiosas. El tatabro no se usa en la medicina tradicional como cura de enfermedades; según los pobladores éste no presta ningún beneficio como analgésico, cicatrizante, ni como estimulante hormonal, es decir, que ninguna de sus partes se utiliza en estas actividades, pero creen que la actividad lunar tiene mucha influencia sobre la movilidad de esta especie, pues suelen cambiar de ruta en luna llena.

El tipo de cacería que se realiza en estas comunidades, es de subsistencia, realizada de forma eventual, lo que quiere decir que no hay dedicación de tiempo completo a esta actividad, por esto durante su desarrollo, las personas no discriminan en capturar machos o hembras, juveniles o adultos, grande o pequeños, solo aprovechan el momento. Zapata-Rios et al. (2006), afirman que estos factores han afectado mucho, en forma negativa, las poblaciones locales de ciertas especies como Tayassus pecari y Tayassus tajacus, entre otras.

\section{CONCLUSIONESYRECOMENDACIONES}

El tatabro se considera como una importante fuente proveedora de proteína animal para las comunidades. En cuanto a su presencia en las zonas de estudio, los cazadores identifican una disminución en el tamaño de las poblaciones de estos animales y una reducción en la frecuencia de captura, que se refleja en la disminución de la cacería de esta especie.

Aún se conservan los métodos de cacería tradicionales como perro, escopeta, lazo, el hacha y el machete, así como otras artes y métodos de cacería; sin embargo, la escopeta en algunas localidades se reemplazó por versiones más modernas con el fin evitar accidentes.

El uso de la fauna silvestre está muy relacionado con el conocimiento empírico y tradicional de las comunidades locales. De igual manera, permite conocer las condiciones económicas, sociales, históricas e identificar las concepciones de los habitantes, lo que es indispensable para la creación de alternativas de manejo para la zona.
A pesar de las acciones o actividades realizadas por instituciones en pro de la conservación de las especies en estudio, se observa un bajo conocimiento por parte de la población, de las problemáticas actuales y las causas que generan perjuicios a la existencia de esta especie, por lo que se requieren acciones educativas que permitan informar, concientizar y aumentar el conocimiento sobre esta especie y su manejo, aplicando estrategias ampliamenteconocidas en el ámbito de la educación ambiental, como la multiplicación, capacitación y difusión de la información, que podría darse desde las escuelas, involucrando a estudiantes, profesores y comunidad educativa en este proceso.

\section{AGRADECIMIENTOS}

A la Corporación CODECHOCO y al Instituto de Investigaciones Ambientales del Pacífico (IIAP), por el apoyo brindado para llevar a feliz término el estudio; a Paola Cano por su ayuda en logística; a los consejos comunitarios y habitantes de los seis municipios, por su colaboración en campo y por compartir sus conocimientos y experiencias sobre la fauna objeto de estudio.

\section{LITERATURA CITADA}

CODECHOCO-UTCH. 2009. Plan de manejo y conservación de la guagua (Cuniculus paca) y el chigüiro (Hydrochoerus isthmius) en los ecosistemas del Medio y Bajo Atrato, Chocó-Colombia. Quibdó: CODECHOCOUTCH; $156 \mathrm{pp}$.

Geilfus, F. 1998. 80 herramientas para el desarrollo participativo: diagnósticos, planificación, monitoreo, evaluación. San Salvador: IICA-GTZ. $208 \mathrm{pp}$.

Guerreros, S., L, Ballesteros. 2006. Caracterización socioculturalyetnozoológica de la población asentada en la cabecera municipal de Quibdó, ChocóColombia. Tesis de trabajo de grado como requisito parcial para optar al título de trabajadora social. Quibdó: Universidad Tecnológica del Chocó «Diego Luis Córdoba». 128 pp.

Morales-Jiménez, Q.L., Sánchez F., K. Poveda, A. Cadena. 2004. Mamíferos terrestres y voladores de Colombia. Guía de campo. Bogotá, DC: quién publica?. $248 \mathrm{pp}$.

Mancera-Rodríguez, N. J., O. Reyes-García. 2008. Comercio de fauna silvestre en Colombia. Rev Fac Nal Agr. 61 (2): 4618-45.

Ojasti, J. 1993. Utilización de la fauna silvestre en América Latina: situación y perspectivas para un manejo sostenible. Roma: Guía FAO de Conservación 25. $248 \mathrm{pp}$.

Ojasti, J. 2000. Manejo de fauna silvestre neotropical. F. Dallmeier (ed.). SI/ MAB Series \# 5. Washington, DC: Smithsonian Institution/MAB Biodiversity Program; 309 pp.

Tejada, E. R., Gómez, H., Painter, L., Wallace R. 2006. Evaluación sobre el uso de la fauna silvestre en la tierra comunitaria de origen tacana, Bolivia. Rev Ecol. 41(2): 138-48.

Valderrama-Ballesteros L. 2009. El saíno (PECARI TAJACU, LINNEUS, 1758) a partir del conocimiento local y la observación de sus huellas, en el territorio de El Valle, Chocó. Bogotá, DC: Pontificia Universidad Javeriana, Programa Académico Ecología; 7 pp.

Zapata-Ríos, G., Araguillin E., J. P. Jorgenson. 2006. Caracterización de la comunidad de mamíferos no voladores en las estribaciones orientales de la cordillera del Kutukú, Amazonía ecuatoriana. Rev Mastozool Neotrop. 13: 227-38 